\title{
Measurement of the electro-optic
}

coefficient during the photoelectric-field assisted poling using a Mach-Zehnder interferometer

Cite as: Rev. Sci. Instrum. 87, 123102 (2016); https://doi.org/10.1063/1.4969057

Submitted: 11 August 2016. Accepted: 15 November 2016. Published Online: 06 December 2016

Flávio M. Shimizu (D), and José A. Giacometti

\section{ARTICLES YOU MAY BE INTERESTED IN}

Mid-infrared gas absorption sensor based on a broadband external cavity quantum cascade laser

Review of Scientific Instruments 87, 123101 (2016); https://doi.org/10.1063/1.4968041

Modulation axis performs circular motion in a $45^{\circ}$ dual-drive symmetric photoelastic modulator

Review of Scientific Instruments 87, 123103 (2016); https://doi.org/10.1063/1.4971302

Characterization of electrical noise limits in ultra-stable laser systems

Review of Scientific Instruments 87, 123105 (2016); https://doi.org/10.1063/1.4971852

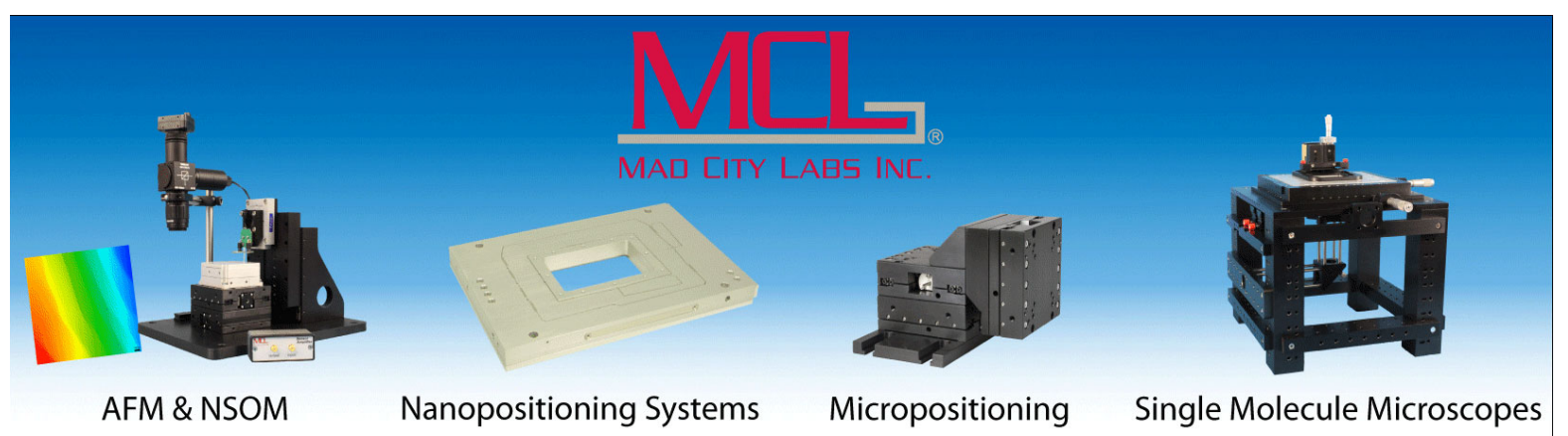




\title{
Measurement of the electro-optic coefficient during the photoelectric-field assisted poling using a Mach-Zehnder interferometer
}

\author{
Flávio M. Shimizu ${ }^{1}$ and José A. Giacometti ${ }^{2}$ \\ ${ }^{1}$ Faculdade de Ciências e Tecnologia, Unesp, 19060-900 Presidente Prudente, São Paulo, Brazil \\ ${ }^{2}$ Instituto de Física de São Carlos, USP, 13566-590 São Carlos, São Paulo, Brazil
}

(Received 11 August 2016; accepted 15 November 2016; published online 6 December 2016)

\begin{abstract}
This work describes the experimental apparatus based on the Mach-Zehnder interferometer for measuring the electro-optic coefficient during and after the photo-assisted poling process using a continuous light excitation. We also show that the poling can be performed at cryogenic temperatures. The setup is based on the superposition of a Mach-Zehnder AC modulation voltage on the DC bias voltage required to promote the chromophore orientation during light excitation. The effect of the reversible polarization due to the DC bias voltage applied to the polymer film is considered to calculate the electro-optic coefficient during the photo-assisted poling process. The usefulness of our setup is demonstrated with three polymer films, with different electro-optic activities: guest-host, copolymer, and homopolymer, all based on methyl methacrylate monomers and containing disperse red-1 chromophores. Published by AIP Publishing. [http://dx.doi.org/10.1063/1.4969057]
\end{abstract}

\section{INTRODUCTION}

Azopolymers are attractive for application in diffraction gratings, data storage, and electro-optical modulators, mainly because of their versatility and low-cost fabrication. ${ }^{1-6}$ For some of these applications, a non-centrosymmetric structure is required to yield the second-order non-linear optical (NLO) activity to the polymer, i.e., chromophore dipoles must have a preferred orientation. Three basic procedures are used to orient the chromophore molecules, viz., thermal assisted poling (TAP) ${ }^{7}$ photo-assisted poling (PAP) ${ }^{8}$ and all optical poling(AOP) ${ }^{9,10}$ In TAP the azo molecules are oriented in a static electric field at temperatures near the glass transition temperature $\left(T_{g}\right)$, and the orientation is frozen by cooling the film down to room temperature with the electric field applied. Heating azopolymer films causes the electrical conductivity to increase near $T_{g}$, which weakens the poling field and an electric film breakdown frequently occurs. To obviate such difficulties, one can employ $\mathrm{PAP}^{8}$ or $\mathrm{AOP}^{9}$ techniques that can be performed at room temperature. ${ }^{11,12}$ AOP is the costliest requiring a pulsed laser with a phase-matched crystal for frequency doubling. PAP is a low-cost alternative requiring a standard $\mathrm{CW}$ laser suitable for the continuous light excitation chromophores to promote reversible trans-cis-trans photoisomerization, thus enhancing the mobility of azo groups to allow the poling to occur under a moderate DC electric field even at low temperatures.

Second order NLO properties can be evaluated by means of the electro-optical (EO) activity, usually measured with Mach-Zehnder interferometer $(\mathrm{MZI})^{13,14}$ or Fabry-Pérot ${ }^{15}$ interferometer, second harmonic generation (SHG), ${ }^{16}$ and reflection techniques. ${ }^{17,18}$ Among these techniques, the most attractive is the Mach-Zehnder interferometer (MZI) as it does not need a pulsed laser required for SHG measurements. So far the MZI has been used to measure the EO coefficient only after the poling process, and therefore the poling information is lost during chromophores orientation.
In this paper, we describe the experimental apparatus in which the PAP technique is coupled with a MachZehnder interferometer for measuring the electro-optic $r_{13}$ coefficient during and after the poling process. Our PAP setup uses the MZI AC modulation superposed on the DC voltage to orient the chromophores. The contribution from the MZI AC modulation voltage and the reversible effect of the DC bias voltage are discussed based on data for poly(methyl methacrylate) containing disperse RED1 dye films, in which we show that poling can be performed at cryogenic temperatures. The adequacy of the setup is further illustrated with determination of $r_{13}$ coefficient during the PAP poling as well as after the poling for two other azobenzenecontaining polymers.

\section{EXPERIMENTAL}

The experimental setup comprises three parts: (i) the Mach-Zehnder interferometer, (ii) the $\mathrm{Ar}^{+}$argon laser to induce continuous reversible trans-cis-trans photoisomerization of dyes, and (iii) the circuitry to apply the DC and AC superposed voltages to the polymer film. The measurement of EO coefficient $r_{13}$ in films is based on the traditional MZI setup described elsewhere, ${ }^{13,19}$ whose schematic diagram is shown in Fig. 1. It uses a low power $1 \mathrm{~mW} \mathrm{He}-\mathrm{Ne}$ as a light source (Coherent-632.8 nm), non-polarizing beamsplitters cubes, and mirrors with high optical quality (Edmund Optics). In one arm of MZI, a mirror with a piezoelectric actuator is used to change the light optical phase with a sawtooth voltage (Stanford DS340) at a frequency of 10 $\mathrm{mHz}$. The film is placed in the other MZI arm for its EO coefficient $r_{13}$ to be measured under an AC modulation voltage at $2 \mathrm{kHz}$ (Agilent 33210A). Neutral variable absorbers are used to control the laser light beam intensities to have the maximum fringe contrast. The polarized light beam at $488 \mathrm{~nm}$ from the $\mathrm{Ar}^{+}$laser (INOVA 70C, Coherent, $488 \mathrm{~nm}$ ) induces 


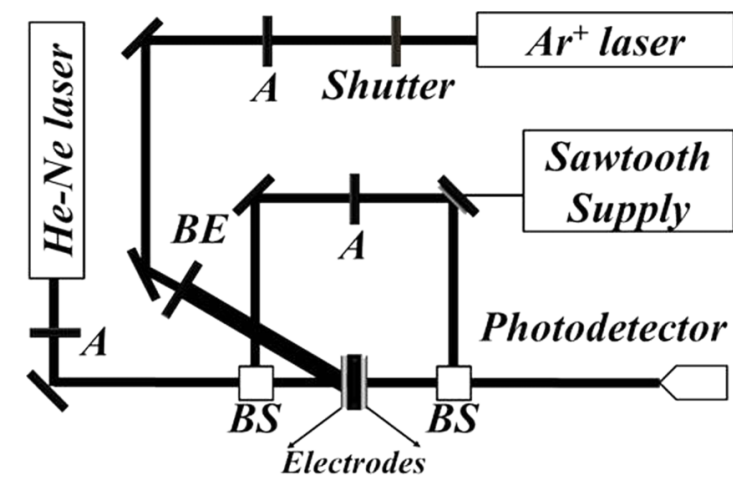

FIG. 1. Photo-assisted poling setup with the schematic drawing of the MachZehnder interferometer. The light from the $\mathrm{Ar}^{+}$laser produces a continuous trans-cis-trans photoisomerization of chromophores. BS are the cube beam splitters and A are neutral absorbers.

the trans-cis-trans photoisomerization of chromophores in the film. The beam expander gives a laser light spot with $1 \mathrm{~cm}$ diameter, and a shutter controls whether the $\mathrm{Ar}^{+}$light reaches the film. The light intensity from the $\mathrm{Ar}^{+}$laser can be varied from 25 to $150 \mathrm{~mW} / \mathrm{cm}^{2}$. The sample with semitransparent electrodes was mounted in the sample holder inside a sealed cryostat (Advanced Research Systems), in order to perform measurements from cryogenic temperature up to $70^{\circ} \mathrm{C}$ under $1 \times 10^{-5} \mathrm{hPa}$ vacuum. Using the same optical parts, a Michelson interferometer was also mounted to evaluate sample deformation under the AC and DC voltages.

To measure the $r_{13}$ coefficient during the PAP process, the DC bias voltage is superposed on the MZI AC modulation voltage by means of the circuitry in Fig. 2. The superposed voltage is produced using a conventional clamper circuit and a high-voltage amplifier (TREK-610C-100x) allowing DC voltages up to $\pm 1 \mathrm{kV}$. This clamping circuit is used to produce the DC electric field required to orient the azo molecules along the film thickness during the continuous reversible trans-cistrans photoisomerization of chromophores.

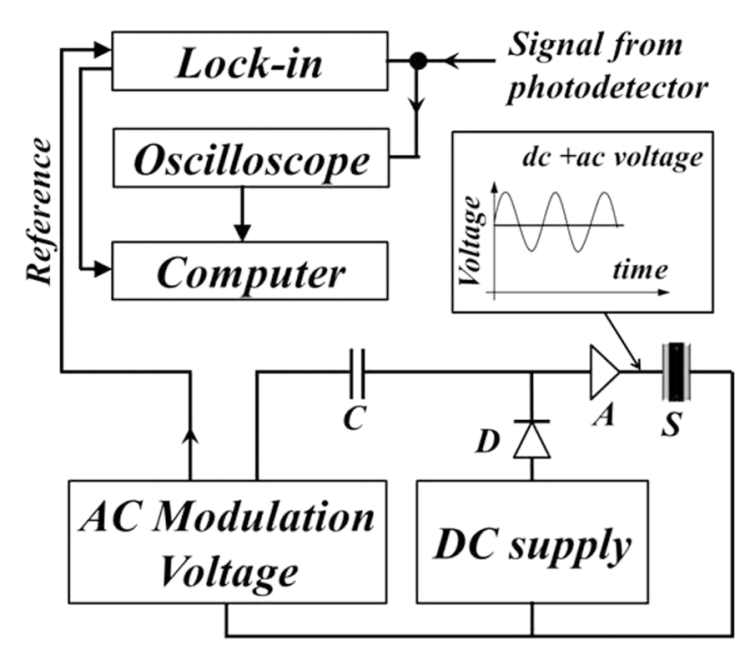

FIG. 2. Electric circuit to obtain the DC and AC superposed voltage. C is a $1.85 \mu \mathrm{F}$ capacitor, $\mathrm{D}$ is the diode $1 \mathrm{~N} 400, \mathrm{~A}$ is the high-voltage amplifier $( \pm 1 \mathrm{kV})$.
The MZI output modulated light is detected by a photodetector and measured using the standard lock-in technique so that the $r_{13}$ EO coefficient can be determined. The light intensity output of the MZI reaching the photodetector due to the EO activity in the film is ${ }^{13}$

$$
I(t)=\frac{I_{\max }-I_{\min }}{2} r_{13} \frac{\pi n_{0}^{3} V_{r m s}}{\lambda} \cos (\omega t) \sin \left(\Delta \phi_{0}\right)
$$

where $\lambda=632.8 \mathrm{~nm}$ is the wavelength of the He-Ne laser, $n_{0}$ is the film refractive index, $V_{r m s}$ is the $r m s$ value of the MZI modulation voltage, and $\omega=2 \mathrm{kHz}$ is its angular frequency. $I_{\max }$ and $I_{\min }$ are the maximum and minimum intensities of the interference fringes giving the fringe contrast. Details on the procedure to determine $r_{13}$ from Eq. (1) are described elsewhere. ${ }^{13,14}$ The phase difference $\Delta \phi_{0}$ is made to vary slowly at $20 \mathrm{mHz}$ using the piezoelectric actuator and the sawtooth voltage supply so that the fringes are moved in front of the photodetector, for $I_{\max }$ and $I_{\min }$ to be determined. The electric signal generated by the photodetector was measured with a lock-in amplifier (Stanford 510) tuned at the frequency $\omega$, and the data are transferred to a microcomputer using a 12 bit data acquisition board. Values of $I_{\max }$ and $I_{\min }$ are obtained directly from the oscilloscope screen (Minipa 2530B).

Three polymer films cast from chloroform solutions were used. One guest-host film of poly(methyl methacrylate) (PMMA), containing 5 wt. \% of DR1 dye (PMMA/DR1). The second was the homopolymer of methyl methacrylate with the DR1 group laterally attached to the monomer (PMDR1). The third one is the copolymer containing monomers of methyl methacrylate (85\%) and methyl methacrylate with DR1 (15\%) laterally attached to the monomer (PMMAcoMDR1). All the polymers were purchased from Aldrich. Drop-cast films were fabricated onto transparent conductive ITO substrates $(50 \Omega)$ and dried in a vacuum oven at $40^{\circ} \mathrm{C}$. Semitransparent aluminum electrodes, $\sim 12 \mathrm{~nm}$ thick, were deposited onto the drop-cast films under high vacuum $\left(\sim 10^{-6}\right.$ Torr) by thermal evaporation (Auto 306 from Boc Edwads).

\section{THE PAP METHOD}

The EO coefficient measured with the MZI technique includes the contribution from the AC modulation voltage, ${ }^{20}$ i.e., values of $\mathrm{EO}$ are overestimated, which is particularly important when the film has a low EO activity. Usually this effect is circumvented using independent experimental techniques ${ }^{21}$ or special analysis of the measured results. ${ }^{22,23}$ Here the contribution of MZI AC modulation voltage is considered and the effect of DC bias voltage in the measured EO signal is also taken into account to estimate the true values of EO coefficients during PAP.

As a proof of concept of the MZI/PAP technique, experiments were performed using a guest-host $12 \mu \mathrm{m}$ thick PMMA/DR1 film. Before each measurement, the neutral absorber in the MZI arm was adjusted to get the maximum fringe contrast, which is around $92 \%$ in most measurements. For PMMA/DR1 the refractive index used in calculations is $n_{0}=1.50 .^{24}$ 

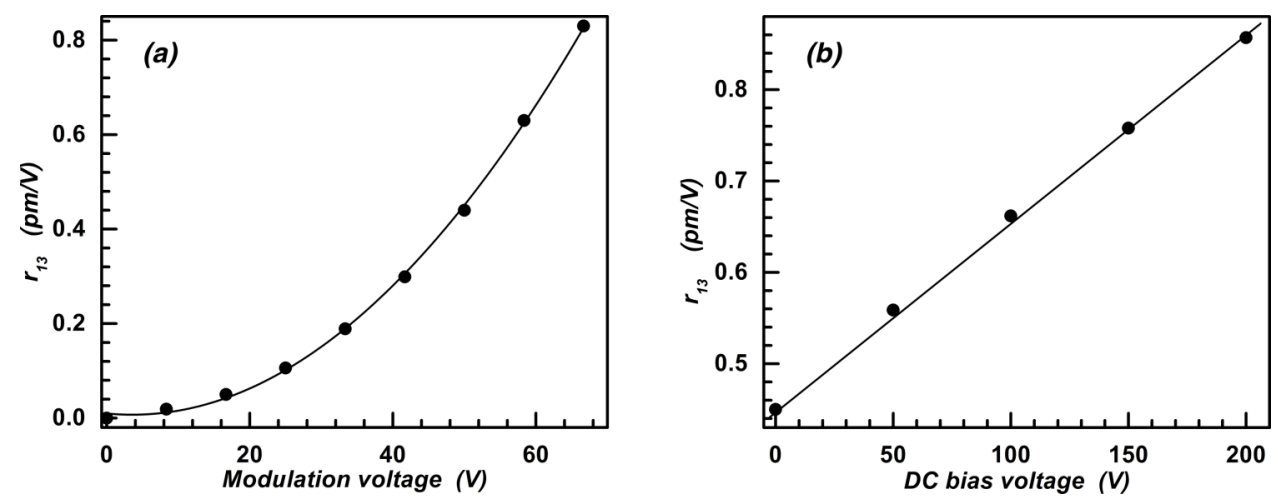

FIG. 3. (a) Dependence of $r_{13}$ on the MZI AC modulation voltage (rms) without applying the DC bias voltage. (b) Dependence of $r_{13}$ on the DC bias voltage for the AC modulation voltage of $50 \mathrm{~V}$. The films used were $12 \mu \mathrm{m}$ thick PMMA/DR1 at room temperature.

\section{A. Effect of AC modulation voltage and DC bias voltage}

The first experiments were carried out on a non-poled PMMA/DR1 film to measure the effect from the AC modulation voltage, $V_{m}$, on the MZ interferometer. Fig. 3(a) shows $r_{13}$ calculated from Eq. (1) versus the AC voltage without applying the DC bias. Therefore, the modulation voltage will induce overestimated values of $r_{13}$. Its contribution to $r_{13}$ is proportional to the squared $\mathrm{AC}$ voltage as inferred from Fig. 3(a). We also found that the DC bias voltage contributes to $r_{13}$ as shown in Fig. 3(b), for the AC modulation voltage of $V_{m}=50 \mathrm{~V}$. In this case, the signal increased linearly with the DC bias voltage. As will be shown, upon removing the DC bias voltage the measured $r_{13}$ practically vanishes, and therefore the effect of the DC voltage is reversible.

The quadratic dependence of $r_{13}$ versus AC voltage in Fig. 3(a) can be explained by the mechanical film deformation due to free charges on the electrodes ${ }^{25}$ rather than being attributed to the piezoelectric effect (small contribution, $d_{33}$ $=0.2 \mathrm{pm} / \mathrm{V}^{26}$ ). This proves that polarization of PMMA/DR1 film is too slow to follow the electric field at the frequency of $2 \mathrm{kHz}$ of the AC voltage. On the other hand, the linear dependence of $r_{13}$ versus DC bias is compatible with changes in the refraction index due to the buildup of a reversible polarization of the PMMA/DR 1 film, ${ }^{22}$ as reported in the similar results by Zekkat ${ }^{27}$ and Osman. ${ }^{8,17}$

Assuming a constant contribution during and after the PAP process, the effect of the AC modulation voltage can be subtracted from $r_{13}$ values, which corresponds to establishing a baseline to avoid the influence of the AC voltage. It is worth mentioning that in our experiments if the AC/DC amplitude ratio is higher than 0.3 , the efficiency of PAP process decreases, that is, $r_{13}$ becomes dependent on the MZI AC modulation voltage. Thus, photo-assisted poling (PAP) requires a MZI AC modulation voltage as small as possible compared to the bias DC voltage. The reversible effect of applying the DC bias voltage will be discussed in Sec. III B.

\section{B. Measurements during the poling process}

The photo-assisted poling process was performed by applying $100 \mathrm{~V}$ DC bias voltage, corresponding to a moderate electric field of $80 \mathrm{kV} / \mathrm{cm}$. The contribution of the DC bias voltage values of the EO coefficient, hereafter called nominal values, $r_{13}^{n}$, was calculated with Eq. (1) and subtracting the base line due to the MZI AC modulation voltage for $30 \mathrm{~V}$ ( $\mathrm{rms}$ ). Note that the curves in Fig. 4 are the superposition of distinct measurements using only one sample. Reproducible data with a sample are possible since the EO activity in PMMA/DR1 disappears after several hours at room temperature.

Curve I shows the effect of the DC voltage on $r_{13}^{n}$ between 3 and $18 \mathrm{~min}$, without light excitation. The EO coefficient increased before stabilizing at about $5 \mathrm{~min}$ at $r_{13}^{n}=0.22 \mathrm{pm} / \mathrm{V}$. For Curve II, at $18 \mathrm{~min}$, the DC bias voltage was removed and the EO activity decayed nearly to zero within a short period. The fast response is due to mechanical deformation and variation of the refractive index in the polymer film, rather than due to light excitation. The small residual signal indicates that a small quasi-permanent polarization remains in the PMMA/DR1 film and is not due to piezoelectric activity. In Curve III, with light excitation on from 18 to $35 \mathrm{~min}, r_{13}^{n}$ increased up to $0.42 \mathrm{pm} / \mathrm{V}$. During this period, the $\mathrm{Ar}^{+}$light produces the trans-cis-trans photoisomerization of chromophores leading to an increase in their mobility, thus allowing them to orient under the bias voltage at room temperature. In Curve IV, a further increase in $r_{13}^{n}$ up to $0.5 \mathrm{pm} / \mathrm{V}$ occurred when the excitation light was turned off at 35 min under an applied DC bias voltage. A similar increase was observed by Zekkat and $\mathrm{Knoll}^{27}$ and Rodriguez et al. ${ }^{28}$

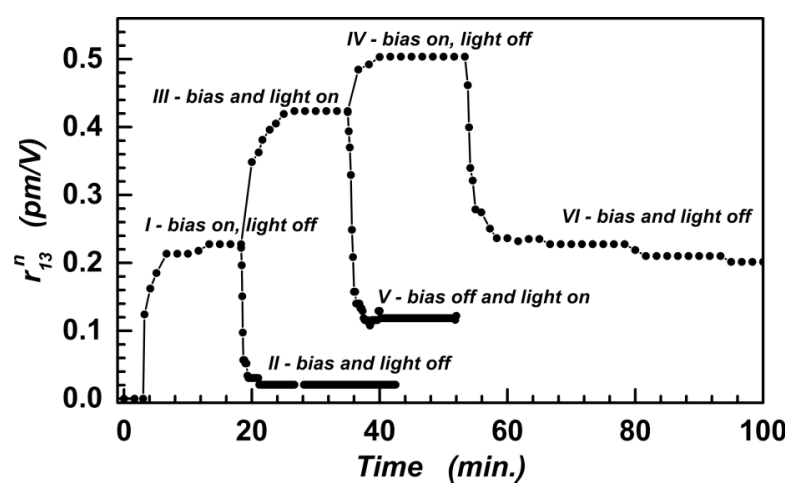

FIG. 4. Curves of the EO activity during PAP for the PMMA/DR1 film. The optical signal from the AC modulation voltage was subtracted. 
which was explained by considering that the dye molecules relax to another equilibrium state depending on the mobility of chromophores. ${ }^{27}$

\section{Decay measurements after the poling}

The decay of $r_{13}$ can be measured under two conditions, as follows. In Curve $V$ in Fig. 4, the DC bias voltage was turned off at 38 min while the light excitation was on, and a fast decay to $r_{13}=0.12 \mathrm{pm} / \mathrm{V}$ was observed since the reversible polarization vanished. As for Curve VI, when the DC bias was turned off at 54 min without light excitation, the EO activity decayed within $\sim 20 \mathrm{~min}$ to $r_{13}=0.22 \mathrm{pm} / \mathrm{V}$. Values of $r_{13}$ in the decay curves $V$ and $V I$ in Fig. 4 are "true values" (the superscript $n$ is omitted) for EO since the DC bias was off and the effect of the AC modulation was already subtracted. Both curves decayed fast when the DC bias was turned off because the reversible effects disappeared. For a long time there is a very slow decay in our films corresponding to the chromophores reorientation. In summary, the maximum residual EO activity can be obtained from curves $I I I$ and VI in Fig. 4, i.e., first the light excitation is turned off under the electric field and then the DC bias is turned off. Furthermore, although a comparison of EO coefficient values is not straightforward because experimental conditions are not the same, values for PMMA/DR1 found in this work are of the same order of those published. ${ }^{25,29}$

\section{Poling dependence on temperature}

Photo-assisted poling can be performed even at cryogenic temperatures, which is advantageous compared to other methods since it does not require elevated temperatures, compatible with the fabrication procedures of photonic devices. Here we successfully achieved the poling of azopolymers films at low temperatures; Fig. 5 shows the maximum nominal values of $r_{13}$ attained at the end of the poling process for the $12 \mu \mathrm{m}$ thick PMMA/DR1 film. The data correspond to the nominal $r_{13}^{n}$ from Curve IV at different temperatures. Similar results were obtained with PMMA/DR 1 films, ${ }^{30}$ where photoinduced birefringence could be achieved at temperatures down to $20 \mathrm{~K}$.

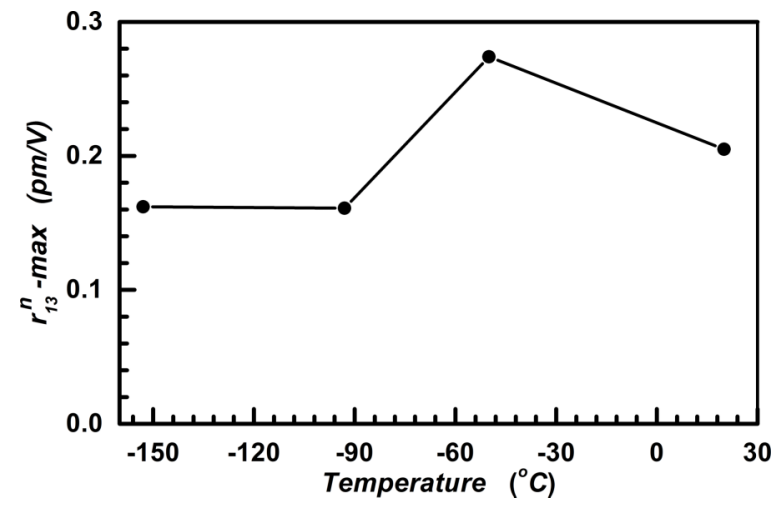

FIG. 5. Maximum nominal values of $r_{13}^{n}$ during PAP for the $12 \mu \mathrm{m}$ thick PMMA/DR1 film at different temperatures.

\section{RESULTS FOR PMDR1 AND PMMACOMDR1}

A guest-host polymer system has a low electro-optic activity. In order to analyze materials with high $r_{13}$, we used azopolymer PMMAcoMDR1 and PMDR1 films, 8 and $4 \mu \mathrm{m}$ thick, respectively. The measurements were taken with different samples since the EO activity lasts for long times (up to 3 weeks); therefore, special care was taken during film fabrication to get the same thickness for all samples. The time dependence of EO activity for the PMMAcoMDR 1 and PMDR 1 films has the same trend shown in Fig. 4 for the guesthost PMMA/DR1 film. It was then omitted. We shall refer to the maximum nominal values $r_{13}^{n}$-max at the end of poling under the same conditions given by Curve IV in Fig. 4. The residual values will be named $r_{13}$-res (true values), taken after 10 min of decay under the same condition of Curve VI in Fig. 4. For the sake of comparison, results for guest-host PMMA/DR1 films are also included in Figs. 6 and 7, where both $r_{13}^{n}$-max and $r_{13}$-res increase linearly with the $\mathrm{Ar}^{+}$light intensity and with the DC bias voltage. As expected, $r_{13}^{n}$ is largest for PMDR1 and smallest for PMMA/DR1 because each monomer of the homopolymer contains one chromophore, while the guest-host PMMA/DR 1 contains only $5 \mathrm{wt}$. \% of DR1. For the copolymer with $15 \%$ of monomers with DR1 laterally attached, $r_{13}^{n}$ is intermediate. Furthermore, the results for $r_{13}^{n}$ versus DC bias voltage in Fig. 6 were obtained with a $4 \mu$ m HEMADR1 film;
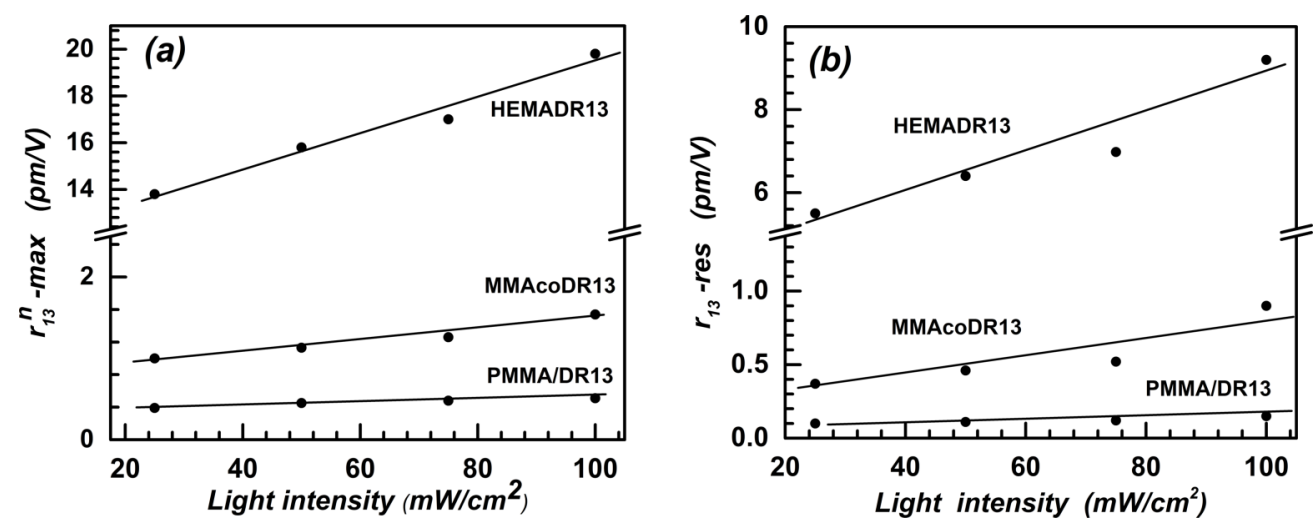

FIG. 6. Dependence of the (a) maximum nominal values $\left(\mathrm{r}_{13}^{\mathrm{n}}\right.$-max) and (b) residual values ( $\left.\mathrm{r}_{13}-\mathrm{res}\right)$ on the Ar + light intensity for PMMA/DR1, PMMAcoMDR1, and PMDR1 films. The lines are for guiding the eyes only. 

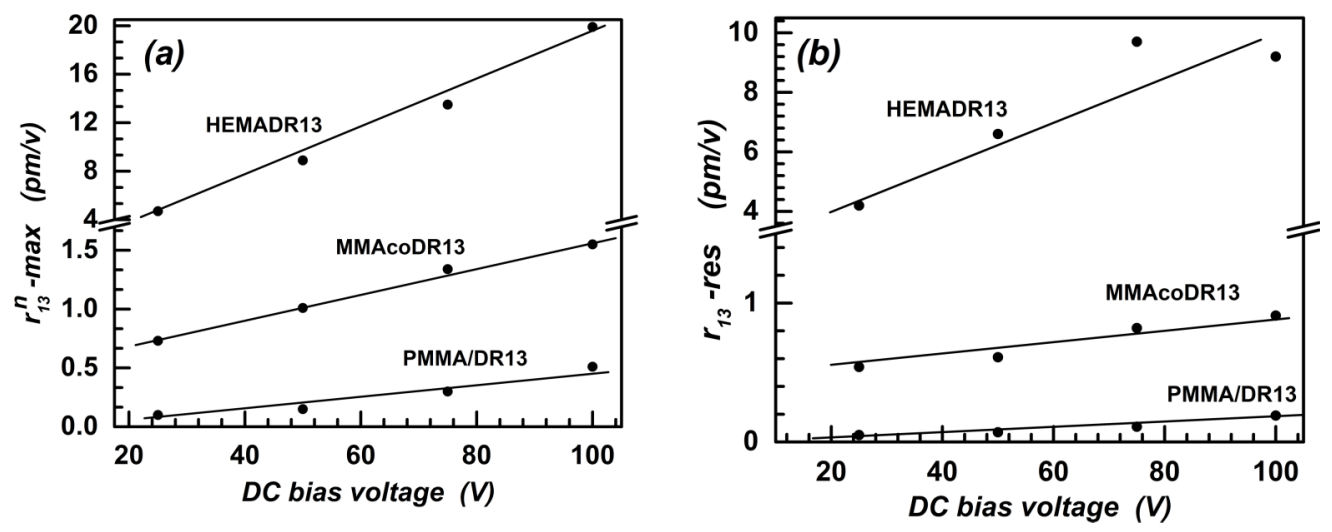

FIG. 7. Dependence of the (a) maximum nominal values $\left(\mathrm{r}_{13}^{\mathrm{n}}\right.$-max) and (b) residual values $\left(\mathrm{r}_{13}\right.$-res) on the DC bias voltage for PMMA/DR1, PMMAcoMDR1, and PMDR1 films. The lines are for guiding the eyes only.
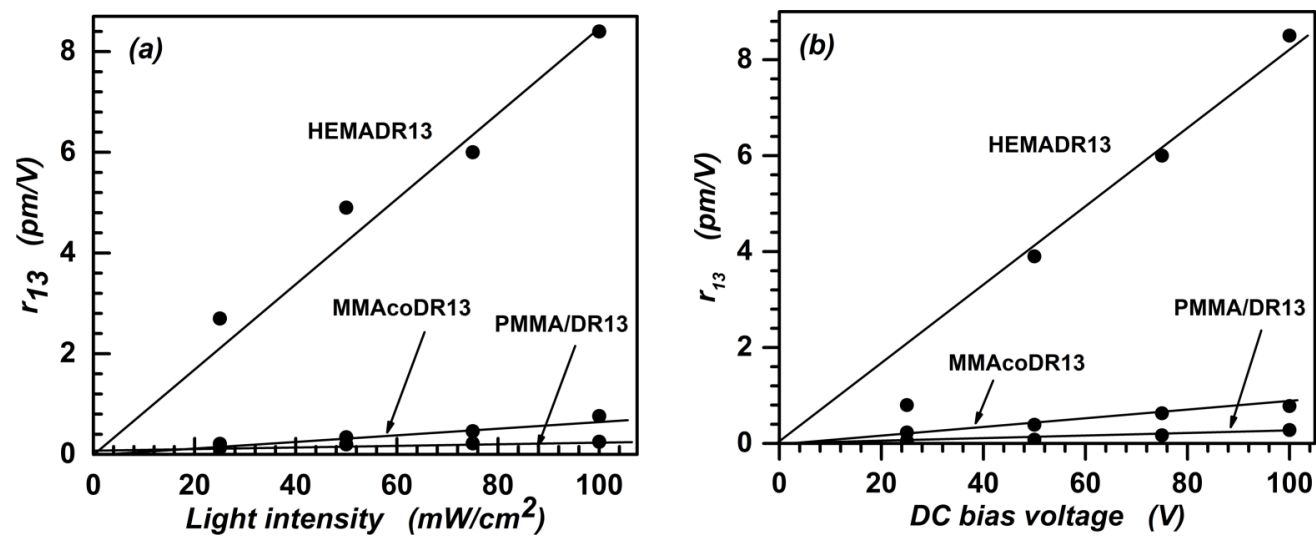

FIG. 8. Dependences of the true maximum values for $\mathrm{r}_{13}$ during the PAP (a) on the light intensity and (b) on the DC bias voltage for PMMA/DR1, PMMcoMDR1, and PMDR1 films. The lines are for guiding the eyes only.

therefore, it was submitted to higher electric fields than the PMMA/DR1 $(12 \mu \mathrm{m})$ and PMMAcoDR1 $(8 \mu \mathrm{m})$ films.

In order to obtain the true values of $r_{13}$ attained during poling, one has to remove the effect from the DC bias voltage. For example, for the PMMA/DR1 film in Fig. 4, the initial jump gives $r_{13}^{n}=0.22 \mathrm{pm} / \mathrm{V}$ while at the end of poling $r_{13}^{n}=0.5 \mathrm{pm} / \mathrm{V}$; therefore, the true value of $r_{13}$ is $0.28 \mathrm{pm} / \mathrm{V}$. The initial jump also occurs for PMMAcoMDR1 and PMDR1 films, and $r_{13}$ decays to zero after removing the DC bias voltage. Figure 8 shows the true maximum values of $r_{13}$ for the films studied here, and the extrapolated values to zero bias and light intensity cross the graphs origin.

The true maximum values of $r_{13}$ for PMMAcoMDR1 and PMDR1 films are larger than the residual values of $r_{13}$ after PAP, which is consistent with the reversible polarization during PAP. In the case of PMMA/DR1 films, the real maximum values of $r_{13}$ are equal or slightly smaller than the residual values. We also recall that after the poling the DC bias is off and does not contribute. The values of $r_{13}$ cannot be compared with the data in the literature in a straightforward manner, since $r_{13}$ depends on experimental conditions, such as excitation light intensity, film thickness, DC bias voltage, and dye content. To our knowledge, for PMMAcoMDR1 and PMDR1 films, there are no published data available.

\section{v. CONCLUSIONS}

The experimental setup presented here is for measuring the electro-optic activity during and after the photo-inducedpoling process applied to the polymer films containing DR1 azobenzene chromophores. The determination of the electrooptic activity during PAP was possible by superimposing the DC voltage to the AC modulation voltage used to run the Mach-Zehnder interferometer. It is concluded that the contribution of the AC modulation voltage can be easily subtracted, and the refractive index changes due to reversible polarization plays an important role to the measured value of the electro-optic coefficient during the PAP for thin films of polymeric materials. The EO activity in three acrylic polymers containing DR1 chromophores were used to show the capabilities of the technique. We showed in Section III D that the PAP poling can be performed even below the room temperature. The PAP technique can be accomplished with a low cost standard lasers and it can be used for producing micropatterns and therefore electro-optic integrated devices.

\section{ACKNOWLEDGMENTS}

The authors acknowledge FAPESP, CNPq, and INEO for the financial support and O. N. Oliveira, Jr. for suggestions in revising the manuscript. 
${ }^{1}$ A. Priimagi and A. Shevchenko, "Azopolymers-based micro- and nanopatterning for photonic applications," J. Polym. Sci., Part B: Polym. Phys. 52, 163 (2014).

${ }^{2}$ J. Liu, G. Xu, F. Liu, I. Kitky, X. Liu, and Z. Zhen, "Recent advances in polymer electro-optic modulators," RSC Adv. 5, 15784 (2015).

${ }^{3}$ O. N. Oliveira, Jr., D. S. Santos, Jr., D. T. Balogh, V. Zucolotto, and C. R. Mendonça, "Optical storage and surface-relief gratings in azobenzenecontaining nanostructured films," Adv. Colloid Interface Sci. 116, 179 (2005).

${ }^{4}$ C. R. Mendonça, A. Dhanabalan, D. T. Balogh, L. Misoguti, D. S. Santos, Jr., M. A. Pereira-Da Silva, J. A. Giacometti, S. C. Zilio, and O. N. Oliveira, Jr., "Optically induced birefringence and surface relief gratings in composite Langmuir-Blodgett (LB) films of poly[4'[[2-(methacryloyloxy)ethyl]ethylamino]-2-chloro-4-nitroazobenzene] (HPDR13) and cadmium stearate," Macromolecules 32, 1493 (1999).

${ }^{5}$ C. R. Mendonça, L. Misoguti, A. A. Andrade, S. B. Yamaki, T. D. Z. Atvars, and O. N. Oliveira, Jr., "Photo-induced birefringence in di-azo compounds in polystyrene and poly(methyl methacrylate) guest host systems," Opt. Mater. 30, 216 (2007).

${ }^{6}$ V. P. Katopodis, A. N. Groumas, Z. Zhang, R. Dinu, E. Miller, A. Konczykowska, J.-Y. Dupuy, A. Beretta, A. Dede, H. H. Choi, P. Harati, F. Jorge, V. Nodjiadjim, M. Riet, G. Cangini, A. Vannucci, N. Keil, H.-G. Bach, N. Grote, H. Avramopoulos, and Ch. Kouloumentas, "Polymer enabled 100 Gbaud connectivity for datacom applications," Opt. Commun. 362, 13 (2016).

${ }^{7}$ P. A. Ribeiro, D. T. Balogh, J. L. C. Fonseca, and J. A. Giacometti, "Chromophore relaxation in a side-chain methacrylate copolymer functionalized with 4-[N-ethyl-N-(2hydroxyethil)]amino-2'-chloro-4'-nitroazobenzene," Macromolecules 37, 2618 (2004).

${ }^{8} \mathrm{Z}$. Sekkat and M. Dumont, "Photoassisted poling of azo dye doped polymeric films at room temperature," Appl. Phys. B 54, 486 (1992).

${ }^{9}$ E. Heydari, E. Mohajerani, and A. Shams, "All optical switching in azo-polymer planar waveguide," Opt. Commun. 284, 1208 (2011).

${ }^{10}$ A. Donval, E. Toussaere, S. Brasselet, and L. Zyss, "Comparative assessment of electrical, photoassisted and all optical in-plane poling of polymer based electrooptic modulators," Opt. Mater. 12, 215 (1999).

${ }^{11} \mathrm{C}$. W. To and K. Y. Wong, "Comparative studies of molecular reorientations in thermal-assisted and photoassisted electric-field poled nonlinear optical polymers," J. Appl. Phys. 100, 073505 (2006).

${ }^{12}$ Z. Sekkat, A. Knoesen, V. Y. Lee, and R. D. Miller, "Influence of the polymer structure on the achievement of polar orientation in high glass transition temperature nonlinear optical polyimides by photo-assisted poling," J. Polym. Sci., Part B: Polym. Phys. 36, 1669 (1998).

${ }^{13}$ K. D. Singer, M. G. Kuzyk, W. R. Holland, J. E. Sohn, S. J. Lalama, R. B. Comizzoli, H. E. Katz, and M. L. Schilling, "Electro-optic phase modulation and optical second-harmonic generation in corona-poled polymer films," Appl. Phys. Lett. 53, 1800 (1988).
${ }^{14}$ Y.-P. Wang, J.-P. Chen, X.-W. Li, J.-X. Hong, and X.-H. Zhang, "Measuring electro-optic coefficients of poled polymers using fiber-optic Mach-Zehnder interferometer," Appl. Phys. Lett. 85, 5102 (2004).

${ }^{15}$ C. A. Eldering, A. Knoesen, and S. T. Kowel, "Use of Fabry-Pérot devices for the characterization of polymeric electro-optic films," J. Appl. Phys. 69, 3676 (1991).

${ }^{16}$ K. D. Singer, M. G. Kuzyk, and J. E. Sohn, "Second-order nonlinearoptical processes in orientationally ordered materials: Relationship between molecular and macroscopic properties," J. Opt. Soc. Am. B 4, 968 (1987).

${ }^{17}$ A. E. Osman and M. Dumont, "Dynamics of photoinduced orientational order of azo-dyes in polymer films," Macromol. Symp. 137, 137 (1999).

${ }^{18}$ C. C. Teng and H. T. Man, "Simple reflection technique for measuring the electro-optic coefficient of poled polymers," Appl. Phys. Lett. 56, 1734 (1990).

${ }^{19} \mathrm{M}$. Sigelle and R. Hierle, "Determination of the electrooptic coefficients of 3-methyl 4-nitropyridine 1-oxide by an interferometric phase-modulation technique," J. Appl. Phys. 52, 4199 (1981).

${ }^{20}$ E. Nitiss, A. Bundulis, A. Tokmakov, J. Busenbergs, E. Linina, and M. Rutkis, "Review and comparison of experimental techniques used for determination of thin film electro-optic coefficients," Phys. Status Solidi A 212, 1867 (2015)

${ }^{21}$ S. Ducharme, J. Feinberg, and R. R. Neurgaonkar, "Electrooptic and piezoelectric measurements in photorefractive barium titanate and strontium barium niobate," IEEE J. Quantum Electron. 23, 2116 (1987).

${ }^{22} \mathrm{~W}$. Hi, Y. J. Ding, and X. Mu, "Electro-optic and electromechanical properties of poled polymer thin films," Appl. Phys. Lett. 79, 3749 (2001).

${ }^{23}$ C. Greenlee, A. Guilmo, A. Opadevi, R. Himmelhuber, R. A. Norwood, M. Fallahi, J. Luo, S. Huang, X.-H. Zhou, A. K.-Y. Jen, and N. Peyghambarian, "Mach-Zehnder interferometry method for decoupling electro-optic and piezoelectric effects in poled polymer films," Appl. Phys. Lett. 97, 041109 (2010).

${ }^{24} \mathrm{~N}$. Tanio and M. Irie, "Refractive index of organic photochromic dyeamorphous polymer composites," Jpn. J. Appl. Phys., Part I 33, 3942 (1994).

${ }^{25}$ H. Ono, T. Kikuchi, and Y. Harato, "Electric-field measurements of complex electro-optic effects in poled polymer," Appl. Phys. B 68, 207 (1999).

${ }^{26}$ F. M. Shimizu, Ph.D. thesis, in Portuguese, São Paulo State University, Brazil, 2012.

${ }^{27}$ A. Zekatt and W. Knoll, "Creation of second-order nonlinear optical effects by photoisomerization of polar azo dyes in polymeric films: Theoretical study of steady-state and transient properties," J. Opt. Soc. Am. B 12, 1855 (1995).

${ }^{28}$ F. J. Rodriguez, C. Sánchez, B. Villacampa, R. Alcalá, R. Cases, M. Millaruelo, and L. Oriol, "Optical anisotropy and non-linear optical properties of azobenzene methacrylic polymers," Polymer 45, 2341 (2004).

${ }^{29}$ T. Iwamura, X. L. Liu, S. Suka, and S. Umegaki, "Phase-perturbation-free measurement of electro-optic effect based on Mach-Zehnder interferometer," Rev. Sci. Instrum. 79, 033101 (2008).

${ }^{30}$ F. F. Algnol, F. F. Dall, O. N. Oliveira, Jr., and J. A. Giacometti, "Influence from the free volume on the photoinduced birefringence in azocompoundcontaining polymers," Macromolecules 39, 4914 (2006). 